livraisons

d'Histoire

de l'Architecture

\section{Livraisons de l'histoire de l'architecture}

$31 \mid 2016$

La source photographique dans la pratique de I'historien de l'architecture

\title{
Photographies et monuments historiques : les fonds photographiques de la Médiathèque de l'architecture et du patrimoine
}

Photographs and registered historic sites in France: photographic funds of the Médiathèque de l'architecture et du patrimoine (MAP)

Fotografie und Denkmal: die fotografischen Bestände der Médiathèque de l'architecture et du patrimoine

Isabelle Gui

\section{(2) OpenEdition}

Journals

Édition électronique

URL : http://journals.openedition.org/lha/618

DOI : $10.4000 /$ /ha. 618

ISSN : $1960-5994$

Éditeur

Association Livraisons d'histoire de l'architecture - LHA

Édition imprimée

Date de publication : 14 juillet 2016

Pagination : 91-101

ISSN : 1627-4970

Référence électronique

Isabelle Gui, «Photographies et monuments historiques : les fonds photographiques de la

Médiathèque de l'architecture et du patrimoine », Livraisons de l'histoire de l'architecture [En ligne],

31 | 2016, mis en ligne le 14 juillet 2018, consulté le 19 avril 2019. URL : http://

journals.openedition.org//ha/618; DOI : 10.4000/lha.618

Ce document a été généré automatiquement le 19 avril 2019

Tous droits réservés à l'Association LHA 


\title{
Photographies et monuments historiques : les fonds photographiques de la Médiathèque de l'architecture et du patrimoine
}

\author{
Photographs and registered historic sites in France: photographic funds of the
}

Médiathèque de l'architecture et du patrimoine (MAP)

Fotografie und Denkmal: die fotografischen Bestände der Médiathèque de

l'architecture et du patrimoine

Isabelle Gui

\section{La Médiathèque de l'architecture et du patrimoine}

1 La Médiathèque de l'architecture et du patrimoine (MAP), créée en 1996, est chargée de collecter, étudier, conserver, communiquer et valoriser les archives et la documentation de l'administration des Monuments historiques. Elle est également responsable d'une des collections photographiques parmi les plus importantes d'Europe. La MAP comprend un département de la photographie ainsi que trois autres départements scientifiques: archives et bibliothèque, documentation des objets et des immeubles protégés au titre des Monuments historiques et Centre de recherche sur les Monuments historiques. Le service est installé sur trois sites. C'est à Charenton-le-Pont, le site principal, que le public est accueilli dans un bâtiment rénové qui a ouvert ses portes en 2014 . Un site Internet ${ }^{1}$ donne $^{-}$ accès à plusieurs bases de données dont Médiathek et Mémoire où est décrite et montrée une partie des fonds photographiques conservés au fort de Saint-Cyr (78) ainsi qu'à Charenton-le-Pont $(94)^{2}$.

Le département de la photographie conserve plusieurs millions d'images. Le fonds des Monuments historiques comporte des vues représentant des édifices, des objets, des vitraux, des peintures murales et des sites archéologiques. Le fonds Guerre se compose de 
prises de vue réalisées durant le premier conflit mondial par le Service photographique et cinématographique de l'Armée (SPCA), sous la double tutelle du ministère de l'Instruction publique et des Beaux-arts et du ministère de la Guerre. La Médiathèque conserve également des fonds concernant plus largement les beaux-arts ainsi que des photographies provenant d'ateliers professionnels de portraits, tels ceux de Nadar, Séeberger ${ }^{3}$, Harcourt et Sam Lévin. Enfin, des photographies d'auteurs photographes proviennent de donations, legs et achats. Si le domaine français est incontestablement le mieux représenté, il existe également un nombre non négligeable de vues réalisées dans des pays étrangers, notamment dans les anciennes colonies françaises. On signalera enfin des dépôts de fonds photographiques provenant de la Société française d'archéologie, de la Bibliothèque nationale de France, de l'université de Paris et de la Réunion des musées nationaux. Au sein de cette collection, les photographies d'architecture représentent plusieurs centaines de milliers de vues dont le noyau originel a été constitué par la commission des monuments historiques ${ }^{4}$.

\section{Origine des fonds photographiques}

Dès l'annonce de l'invention du daguerréotype par Arago en 1839, la commission des monuments historiques ${ }^{5}$ perçoit les avantages de ce nouveau procédé pour ses travaux. Réaliser un daguerréotype est cependant complexe et coûteux. Par ailleurs, c'est une photographie unique qui ne permet pas de produire d'autres images. Cet inconvénient est pallié par le négatif papier, procédé inventé par Talbot en 1840 et perfectionné en 1847 par Blanquart-Évrard. En 1851, la Commission commande à Le Gray, Mestral, Le Secq, Baldus et Bayard, des prises de vue de monuments historiques. De cette commande, appelée la " Mission héliographique », la Médiathèque de l'architecture et du patrimoine conserve 258 vues qui sont les premières inventoriées par la Commission ${ }^{6}$. Cependant, dans les années qui suivent, l'essentiel du budget des Monuments historiques est consacré à la restauration des édifices. Ainsi, lorsqu'en 1872 Séraphin-Médéric Mieusement (1840-1905) propose au ministre, puis à la direction des Beaux-arts, de constituer une collection de photographies des monuments déjà classés et de ceux qui présentent « un intérêt historique architectural, artistique ou pittoresque ${ }^{7}$ », son offre est refusée ${ }^{8}$. La situation évolue toutefois rapidement. Le 7 janvier 1873, la commission des monuments historiques décide de réviser la liste des monuments classés9. Le 21 août suivant, une circulaire envoyée aux préfets précise que des photographies doivent être jointes aux dossiers de classement ${ }^{10}$. En 1874, dans un courrier qu'il adresse à Adolphe Dauphinot (1822-1893), le ministre de l'Instruction publique, des Cultes et des Beaux-arts indique que la Commission «réunit en ce moment les vues photographiques des monuments remarquables de toute la France $»^{11}$. Le 28 mars 1877 Anatole de Baudot présente à la Commission son ouvrage sur la sculpture française accompagné de photographies de Mieusement ${ }^{12}$. Il obtient une mission et une allocation pour poursuivre son travail, en contrepartie desquelles il doit «déposer [dans les archives de la Commission] deux exemplaires de chacune des photographies destinées à la publication ${ }^{13} \%$. La collaboration dès lors régulière de Mieusement et d'autres photographes permet le développement de la collection photographique. 


\section{Les commandes et campagnes photographiques}

4 En 1877, les Monuments historiques commandent à Mieusement des photographies de quatre-vingt-sept édifices pour l'Exposition universelle de $1878^{14}$. Quatre ans plus tard, en 1881, le photographe s'engage auprès du ministre de l'Intérieur et des Cultes à exécuter, sous la direction des architectes diocésains, les vues de cathédrales qui lui seront désignées ainsi que celles des édifices attenants: "Les vues seront prises selon les instructions que me donneront les architectes sur la partie du monument à photographier ${ }^{15}$.» Bien que les clichés appartiennent à l'administration des Cultes, la commission des monuments historiques peut cependant en obtenir des tirages ${ }^{16}$. Elle confie aussi à Mieusement la mission de collecter des photographies auprès de photographes locaux ${ }^{17}$. Une centaine d'entre eux envoie aux Monuments historiques des vues de monuments de la ville ou de la région où ils sont établis ${ }^{18}$. Les archives de la Commission s'enrichissent également des photographies de Paul Robert (1867-1898), gendre de Mieusement, et surtout de Jean-Eugène Durand (1845-1926), sous-chef du bureau des Monuments historiques. Comme son contemporain Mieusement, Durand parcourt la France et réalise des milliers de prises de vue de monuments. Les frères Neurdein, chargés par ailleurs de l'exploitation du fonds photographique du service des Monuments historiques jusqu'à fin 1915, vendent également des photographies à l'administration.

Lors de la première guerre mondiale, les opérateurs de la Section photographique et cinématographique de l'armée (SPCA) réalisent des prises de vue des dommages de guerre causés aux monuments historiques. Entre 1923 et 1942, la société des Archives photographiques d'art et d'histoire exploite le fonds photographique du Service photographique et cinématographique des Beaux-arts et est chargée de le compléter ${ }^{19}$. Emmanuel-Louis Mas, ancien opérateur de la SPCA et photographe du service des Archives photographiques, prend près d'un millier d'images en 1939 avant que l'activité du service ne soit considérablement réduite pendant la seconde guerre mondiale. Dans les années qui suivent, le service des Archives photographiques réalise plusieurs campagnes de prises de vue sur le terrain. À partir des années 1970 cette mission est assurée par l'Inventaire général des richesses artistiques de la France ${ }^{20}$.

\section{Acquisitions d'ensembles constitués}

Des ensembles de photographies, constitués par des photographes professionnels ou amateurs, ont été achetés, donnés ou légués tandis que d'autres ont fait l'objet de dépôts. Parmi les ensembles les plus remarquables réalisés par des professionnels, on signalera tout particulièrement le fonds Atget (1857-1927), dont la MAP conserve quelque 4600 négatifs et près de 1800 tirages de vues d'architecture réalisées à Paris et en région parisienne. On trouve également 8000 clichés et de nombreux tirages du photographe Henri Heuzé (1851-1927), beau-père de l'architecte Henri Deneux (1874-1969). Plusieurs photographes amateurs, qui ont pris des images d'un grand intérêt documentaire, doivent aussi être mentionnés. Félix Martin-Sabon (1846-1933), ingénieur des arts et manufactures, se consacre exclusivement à sa passion pour la photographie et l'architecture à partir de 1885. La MAP conserve 15400 de ses négatifs et 6500 plaques de projection, sans compter sa collection personnelle de tirages réalisée à partir de ses clichés. En 1930, la Société française d'archéologie dépose au service des Archives 
photographiques 14000 négatifs sur verre d'Eugène Lefèvre-Pontalis (1862-1923), historien de l'architecture, archéologue et professeur à l'École des chartes ${ }^{21}$. Les architectes contribuent également jusqu'à aujourd'hui à l'enrichissement des fonds photographiques de la Médiathèque. Au XIX siècle, à côté de ceux qui collectent des photographies, comme Eugène-Emmanuel Viollet-le-Duc (1814-1879) ou Jean-Juste Lisch (1828-1910), d'autres les réalisent eux-mêmes tels Alfred-Nicolas Normand (1822-1909), qui prend ses premiers clichés en 1851 et dont la MAP conserve 1300 photographies réalisées en France à la fin du XIX ${ }^{e}$ siècle; ou encore Jean Trouvelot (1897-1985), architecte en chef des Monuments historiques, auteur d'importantes séries d'images prises en Eure-et-Loir, dans l'Yonne et au château de Vincennes.

\section{Typologie}

7 Les photographies de monuments historiques conservées à la MAP, réalisées ainsi qu'il vient d'être indiqué par des photographes professionnels ou amateurs (historiens de l'art, amateurs d'architecture et/ou de photographie, architectes) peuvent être réparties en trois catégories.

\section{Photographie systématique}

8 En observant les images de Mieusement ou celles de Durand, on constate que ces photographes ont élaboré, au contact des architectes, une véritable méthode de travail. Leurs photographies sont réalisées dans un contexte de plus en plus concurrentiel qui les oblige à répondre précisément à la commande. On peut donc considérer que le corpus d'images conservé à la MAP est très représentatif d'une photographie d'architecture conforme aux attentes des membres de la commission des monuments historiques. Dans la fabrication de ces images, il ne faudrait cependant pas négliger le poids des contraintes budgétaires : pour épargner les deniers publics, le photographe doit fournir un maximum d'informations avec le moins d'images possible. Les vues de trois-quarts comme celle de la façade de la cathédrale de Montauban laissent deviner un long côté. Elles renseignent sur la structure de l'édifice en privilégiant le point d'accès principal tout en rendant compte du développement du monument, mais elles permettent aussi d'économiser une vue latérale ${ }^{22}$ quand celle-ci n'offre que peu d'intérêt ${ }^{23}$ (ill. 1). Pour certains édifices religieux, églises et surtout cathédrales, Mieusement livre un petit lot d'images qui comporte, outre celle qui vient d'être mentionnée, une vue éventuelle du chevet et deux vues intérieures, l'une en direction du chœur et l'autre vers la façade. Ces prises de vue sont éventuellement réalisées depuis des échafaudages ou les tribunes pour éviter au maximum les déformations. Mieusement photographie aussi, s'il y a lieu, les détails les plus intéressants : portes, fenêtres, chapiteaux, bas-relief, vitraux. Le photographe doit comprendre ce qu'il voit pour être capable de restituer l'architecture avec des vues les plus concises possibles. Ces images, parfaitement lisibles, sont de véritables outils de travail pour la Commission. 


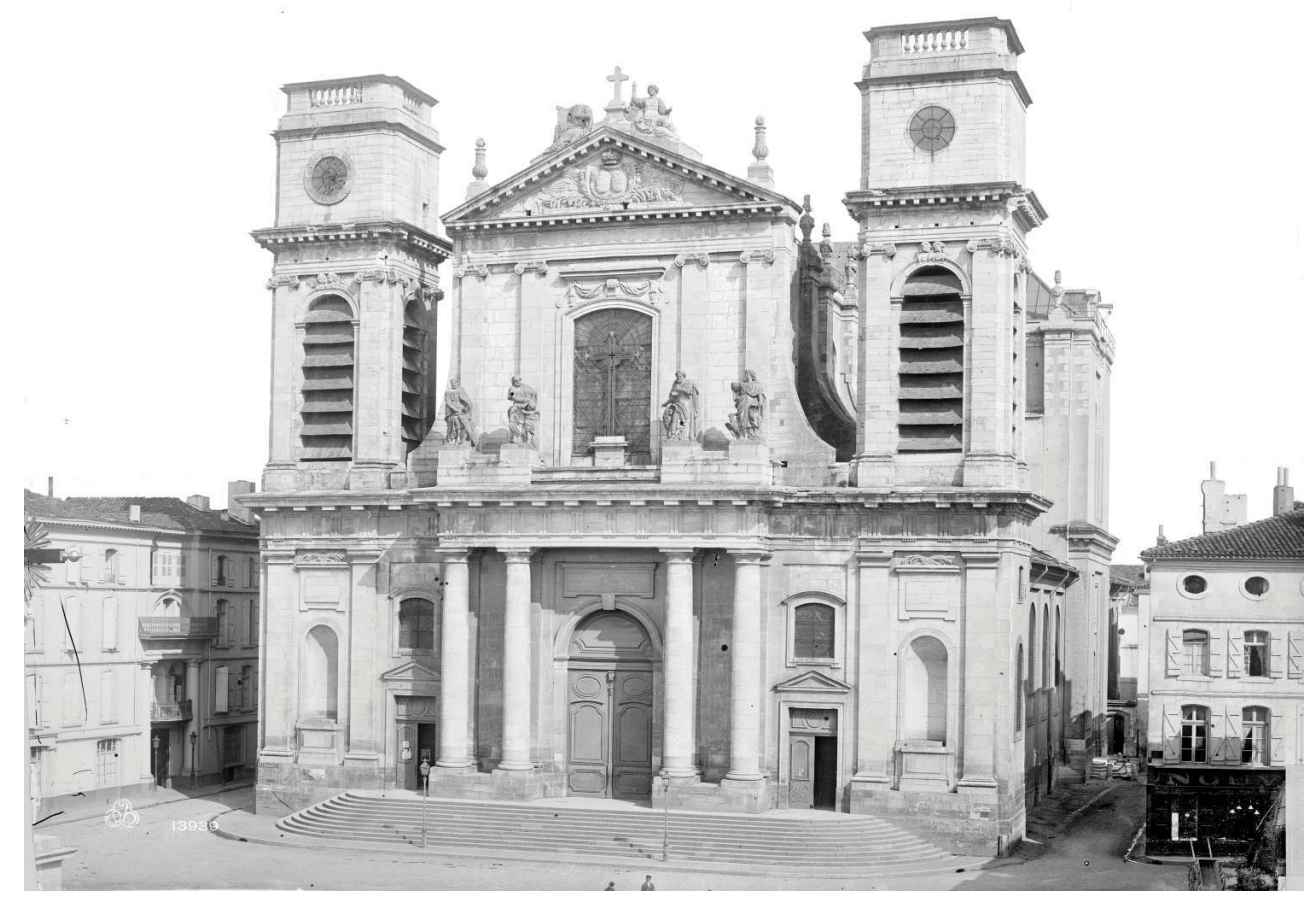

S. d., négatif verre noir et blanc positivé, $30 \times 40 \mathrm{~cm}$, Médiathèque de l'architecture et du patrimoine, $\mathrm{MH} 13939$.

(C) Médiathèque de l'architecture et du patrimoine/dist. RMN-GP

9 La méthode de travail, élaborée durant le dernier tiers du XIX ${ }^{e}$ siècle est reprise en 1917 dans les instructions données aux opérateurs de guerre. Une de leurs missions est en effet de photographier toutes les églises à proximité du front ${ }^{24}$ dans la perspective de leur probable destruction ou pour les faire classer :

«Messieurs les opérateurs [...] voudront bien, en principe, prendre dorénavant de chacune [des églises anciennes] les vues suivantes: a) Extérieur: la façade principale (en général façade ouest), les deux façades latérales (façades nord et sud) et, s'il y a lieu, le chevet. Un des clichés au moins des façades latérales devra, toutes les fois que cela sera possible, être pris de façon à comprendre dans la photographie les parties hautes de l'église (clochers et toitures). b) Intérieur ; la nef et le chœur. À ces clichés d'ensemble devront être joints, s'il y a lieu, des détails du monument portant sur les parties les plus intéressantes et caractéristiques: portails des façades, sculptures, détails des voûtes ou des charpentes, objets mobiliers anciens, etc. ».

\section{Photographie aide-mémoire}

10 Si les prises de vue systématiques telles qu'elles étaient réalisées par les photographes du XIX ${ }^{e}$ siècle n'ont pas disparu aux siècles suivants, l'évolution du matériel photographique permet l'apparition d'une photographie aide-mémoire.

11 L'architecte utilise désormais son propre appareil. Seules ou avec des prises de notes, les photographies qu'il réalise sont autant de matériaux qui lui permettent d'élaborer un projet de restauration. Ce sont des images très personnelles dont la vocation première n'est pas de convaincre la commission des monuments historiques du bien-fondé d'un 
projet de restauration, mais plutôt de permettre à l'architecte de collecter des informations qui accompagnent sa réflexion. À côté de plans larges, de vues qui rendent compte de l'état d'un monument et des travaux qui y sont accomplis, Sylvain StymPopper (1906-1969), architecte en chef des Monuments historiques, prend quantité de photographies de détails (ill. 2). Ce sont précisément ces images de détails qui sont de plus en plus présentes dans les fonds d'architectes, notamment depuis l'apparition de la photographie numérique.

III. 2 : Sylvain Stym-Popper, Entourage de fenêtre du palais des rois de Majorque à Perpignan, juin 1957

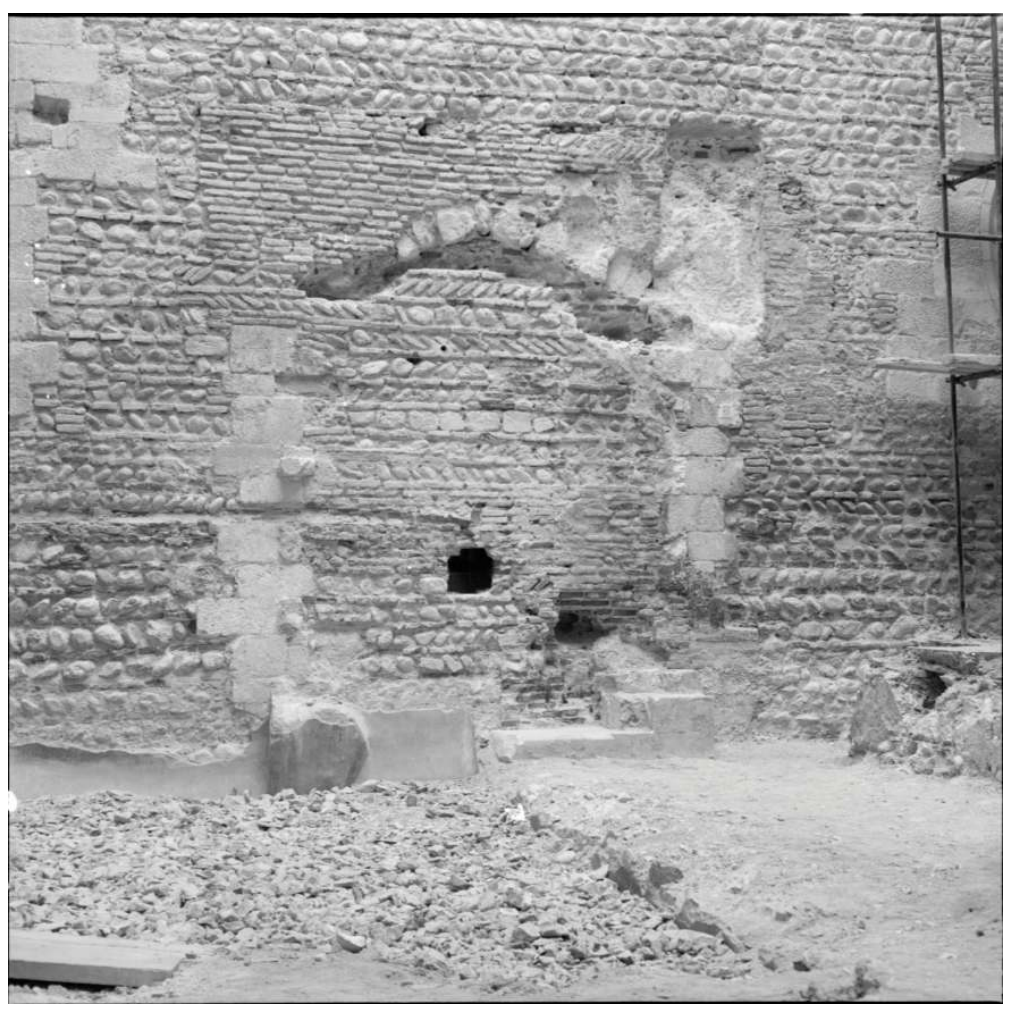

Juin 1957, négatif souple noir et blanc positivé, 6 × $6 \mathrm{~cm}$, Médiathèque de l'architecture et du patrimoine, 44L6944.

(c) Médiathèque de l'architecture et du patrimoine/dist. RMN-GP

\section{Photographie liée à des circonstances particulières}

La MAP conserve depuis le XIX ${ }^{e}$ siècle de nombreuses prises de vue réalisées dans des circonstances particulières : incendies, bombardements, restaurations, reconstructions, voire constructions d'édifices aujourd'hui protégés au titre des Monuments historiques.

Jean-Eugène Durand photographie ainsi le château de Sillé-le-Guillaume (Sarthe) dont les toitures ont été bâchées après un incendie. G. Verneau réalise des prises de vue de la cathédrale de Reims, notamment des voûtes qui ont beaucoup souffert des bombardements de la première guerre mondiale (ill.3). Il photographie également la construction d'une couverture provisoire sur ce même édifice. Quant à A. Masson, il prend vers 1895 quelques vues spectaculaires de la reconstruction de la flèche de la cathédrale Saint-Bénigne de Dijon par l'architecte en chef et architecte diocésain Charles Suisse (1846-1906). Enfin, parmi les 10000 clichés du fonds Henri Deneux (1874-1969), qui 
restaure la cathédrale de Reims à la fin de la première guerre mondiale, on trouve des photographies réalisées entre 1927 et 1929 qui montrent la construction de la halle de Maigrot et Freyssinet à Reims, classée en 1990.

III. 3 : G. Verneau, Vue intérieure de la cathédrale de Reims depuis une brèche ouverte dans les voûtes de la croisée du transept

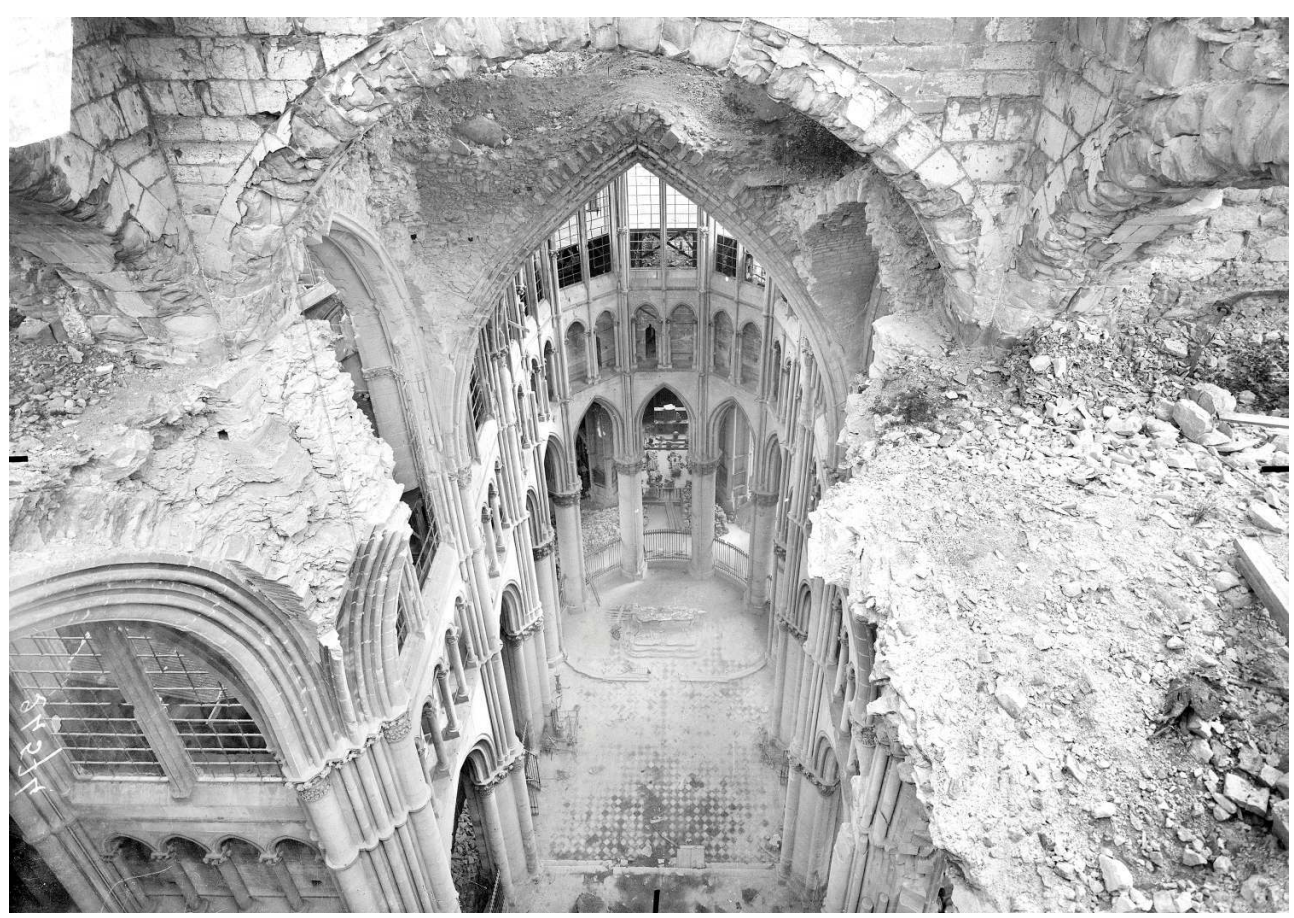

1919, négatif verre noir et blanc positivé, 13 × 18 cm, Médiathèque de l'architecture et du patrimoine, $\mathrm{MH} 24574$.

(c) Médiathèque de l'architecture et du patrimoine/dist. RMN-GP

\section{Pour le public et avec le public}

\section{Documenter un édifice par l'image}

La MAP poursuit l'enrichissement de son fonds de photographies d'architecture par des acquisitions auprès de particuliers et des préemptions en ventes publiques. Les informations disponibles sur cette image (auteur, date, voire circonstances de la prise de vue) et la pertinence du point de vue choisi par le photographe figurent parmi les critères de sélection. Idéalement, l'acquisition d'une photographie doit prendre en compte le public qui recherche le visuel d'un monument à une époque donnée, tout autant que l'historien de la photographie qui sera davantage attentif à la personnalité de l'auteur de cette image.

Un daguerréotype de Stanislas Ratel (1824-1904) et Charles-Marie-Isidore Choiselat (1815-1858) représentant la tour de César à Provins a fait l'objet d'une préemption en vente public en 2015. Cette tour, classée en 1846, a été photographiée en 1851 lors de la Mission héliographique par Henri Le Secq, puis en 1886 par Alfred-Nicolas Normand, entre 1886 et 1896 par Félix Martin-Sabon, en 1900 par Henry de Lestrange et encore bien d'autres photographes tout au long du XXe siècle, dont Marcel Bovis. Ce daguerréotype 
constitue désormais la plus ancienne vue dont dispose la MAP puisqu'il fut réalisé en 1845. Cette série de photographies permet de suivre cet édifice sur plus de cent cinquante ans depuis son classement. Dans le cas précis de la tour de César, ces vues prises sous différents angles ne laissent pas apparaître de transformations majeures, contrairement à l'exemple qui suit.

\section{Confronter les sources}

En 1840, l'église de Saint-Père, dans l'Yonne, est classée monument historique. La restauration, d'abord confiée à Tircuit, un architecte d'Avallon, revient en 1841 à Violletle-Duc. Faute d'argent pour poursuivre le chantier, les travaux sont interrompus en $1849^{25}$ . C'est pour cette raison que Viollet-le-Duc n'aurait pu intervenir sur le porche de la façade occidentale qu'il prévoyait de débarrasser de sa couverture en lave, conformément au souhait de Mérimée. Le porche et la façade n'auraient été restaurés qu'entre 1888 et 1898 par Bœswillwald ${ }^{26}$. Les photographies conservées par la MAP infirment toutefois cette chronologie.

Nous retiendrons deux vues. Sur celle que Baldus avait prise en 1851, on voit le porche sur lequel on distingue clairement une toiture, ce qui confirme bien qu'il n'y a pas eu de modification de la couverture jusqu'à cette date. En revanche, sur la photographie de la façade réalisée par Mieusement en juin 1878, le toit du porche a disparu pour être remplacé par une terrasse. Il y a donc eu une intervention sur cette partie de l'édifice entre 1851 et 1878, avant la reprise des travaux par Bœswillwald (ill. 4-5).

\section{4 : Édouard Baldus, Église de Saint-Père (Yonne)}

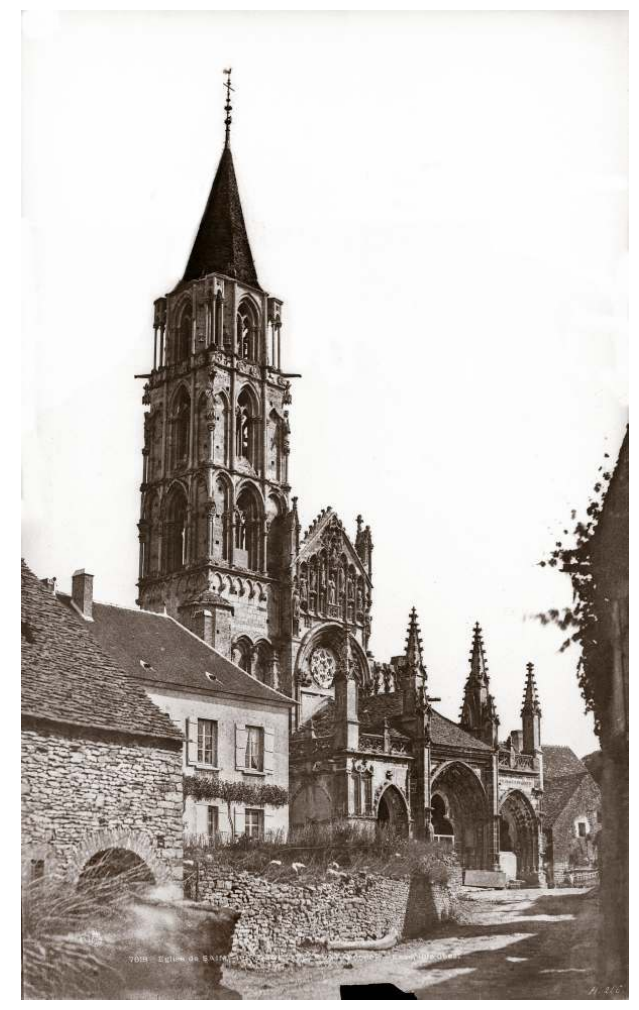

1851, deux négatifs papier noir et blanc assemblés positivés, $44,9 \times 27,5 \mathrm{~cm}$, dépôt de la Médiathèque de l'architecture et du patrimoine au musée d'Orsay, MAP, MH7618, musée d'Orsay, DO 1982499.

(c) Musée d'Orsay/RMN 
III. 5 : Séraphin-Médéric Mieusement, Porche de l'église de Saint-Père (Yonne)

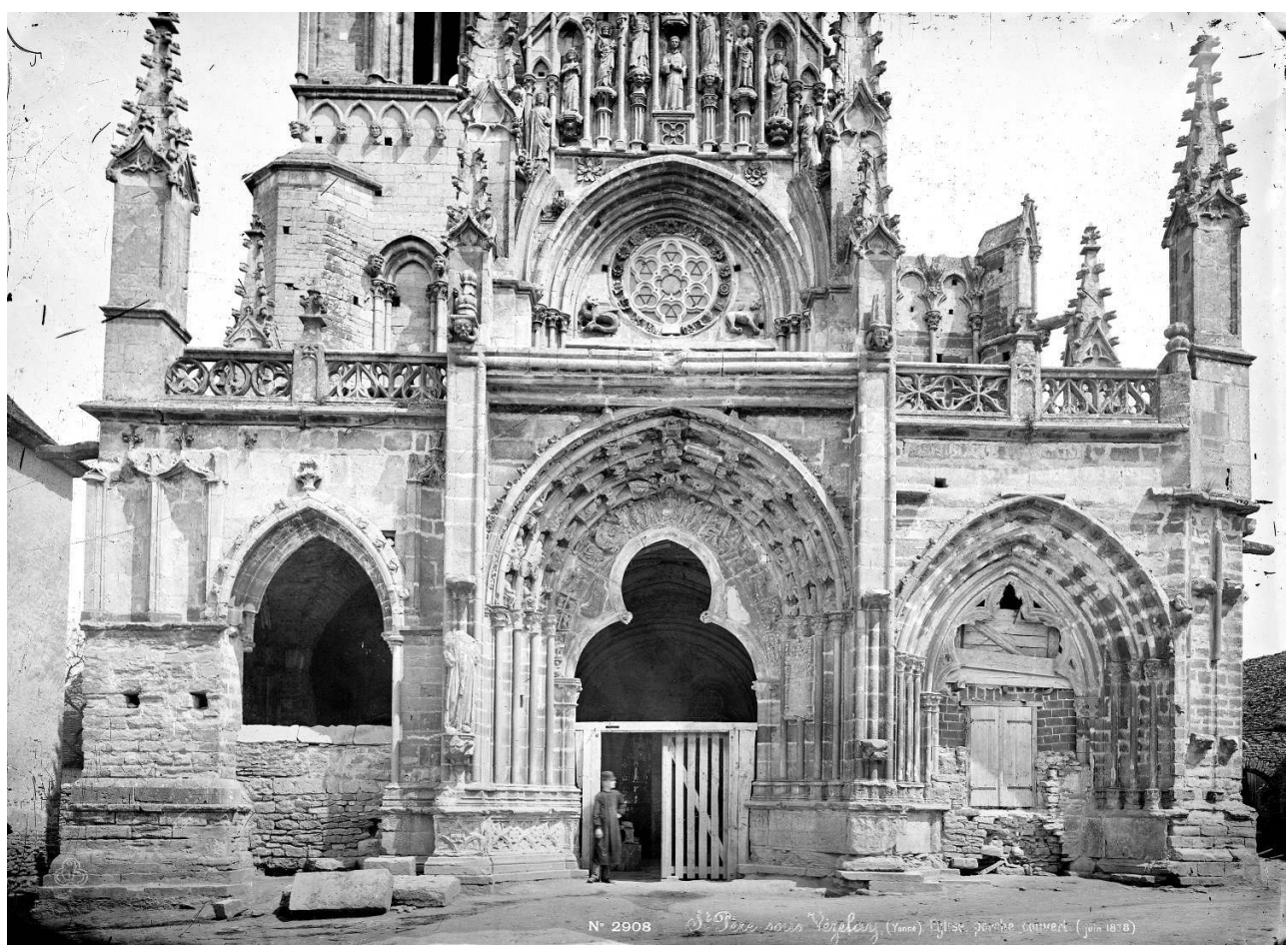

Juin 1878, négatif verre positivé noir et blanc 30 × 40 cm, Médiathèque de l'architecture et du patrimoine, $\mathrm{MH} 2908$.

(c) Médiathèque de l'architecture et du patrimoine/dist. RMN-GP

L'examen conjoint des archives textuelles et des photographies peut ainsi remettre en cause la chronologie des restaurations réalisées sur un monument.

\section{Le rôle du public}

19 L'échange d'informations entre gestionnaires des fonds, historiens de la photographie, historiens de l'architecture et tous ceux qui, pour des raisons d'ordre privé ou professionnel, s'intéressent à un monument est tout à fait essentiel pour compléter la description des images. Il existe en effet dans les fonds de la MAP un grand nombre de photographies non datées et anonymes.

Des vues du château de Fresney-Le-Puceux dans le Calvados ont pu être datées d'avril ou mars 1930, année même du classement, grâce à un lecteur qui connaissait parfaitement l'histoire du monument. En revanche, l'auteur de cette vue reste pour le moment inconnu.

Une photographie d'un détail de l'église de Lanleff (Côtes-d'Armor), datée de 1872, a pu être attribuée à Jules de Laurière, qui fut secrétaire de la Société française d'archéologie. Il prit vraisemblablement cette photographie lors de sa visite du monument le 5 juillet 1872, pendant le Congrès scientifique de France réuni à Saint-Brieuc ${ }^{27}$. Un lieu, un nom, une date, un contexte de prise de vue : l'information est désormais complète pour une photographie témoignant de l'intérêt porté à cet édifice qui ne fut classé qu'en 1889. 


\section{NOTES}

1. Site Web de la Médiathèque de l'architecture et du patrimoine, URL: http :/ www.mediatheque-patrimoine.culture.gouv.fr, dernièrement consulté le 15 mars 2016. L'architecture informatique de ce site a été conçue par notre collègue Jean-Luc Pouvesle (1956-2015).

2. Situé sur la commune de Montigny-le-Bretonneux.

3. Les fonds Nadar, Séeberger et Atget, dont il sera question plus loin, sont classés au titre des Monuments historiques.

4. Pour davantage de précisions sur l'histoire de la constitution de ces fonds, on se référera à l'ouvrage d'Anne Fourestié et Isabelle Gui à paraître fin 2016.

5. MAP, 80/1/17, rapport de Jean Vatout, président du Conseil des Bâtiments civils au ministre, secrétaire d'État au département de l'Intérieur, portant "proposition de nommer une commission pour l'examen des travaux à faire aux Monuments historiques ».

6. Voir l'ouvrage d'Anne de Mondenard, La Mission héliographique : cinq photographes parcourent la France en 1851, Paris, Monum, Éd. du patrimoine, 2002, $320 \mathrm{p}$.

7. MAP, 80/1/73, lettre de Mieusement au ministre des Beaux-arts, 22 juin 1872.

8. MAP, 80/1/73, minute de lettre du directeur des Beaux-arts à Mieusement, 23 juillet 1872.

9. Jean-Daniel Pariset. Procès-verbaux de la Commission des monuments historiques conservés à la Médiathèque de l'architecture et $d u$ patrimoine. URL: http://elec.enc.sorbonne.fr/ monumentshistoriques/, dernièrement consulté le 15 mars 2016.

10. En 1848, la Commission des Arts et édifices religieux avait demandé que les projets de restauration soient accompagnés de daguerréotypes: Archives nationales, F19/4536, Instruction pour la rédaction des projets, l'exécution des travaux et la rédaction des mémoires concernant les édifices religieux, préparée par la commission instituée, par arrêté du 7 mars 1848, près la direction générale de l'administration des Cultes, 25 juillet 1848. Imprimerie nationale, 1848, $6 \mathrm{p}$.

11. MAP, 80/74/1, lettre du ministre de l'Instruction publique, des Cultes et des Beaux-arts à Adolphe Dauphinot, président de la Société des amis des arts de Reims, 24 octobre 1874.

12. La Sculpture française au Moyen Âge et à la Renaissance, Paris, Des Fossez et C ${ }^{\mathrm{ie}}, 1884,40$ p. et [97] pl.

13. Jean-Daniel Pariset, op. cit.

14. MAP, 80/74/66, lettre de Mieusement, 17 juillet 1877.

15. MAP, 80/13/71, soumission, 11 avril 1881.

16. MAP, $80 / 1 / 73$, lettre du ministre de la Justice et des Cultes au ministère de l'Instruction publique et des Beaux-arts, 13 mars 1883. La collection sera affectée aux Monuments historiques après la loi de séparation des Églises et de l'État

17. MAP, 80/74/66, minute de lettre du secrétaire de la commission des monuments historiques à Mieusement, 7 janvier 1876.

18. MAP, $80 / 74 / 1$, liste de MM. Les photographes en relation avec l'administration des Monuments historiques, s.d.

19. MAP, 80/74/5, contrat passé entre le ministre de l'Instruction publique et des Beaux-arts et Henri Thénot représentant les Archives photographiques d'art et d'histoire, 3 janvier 1923, article 14 .

20. Créée par le décret $n^{\circ}$ 64-203 du 4 mars 1964.

21. MAP, 80/74/30, convention passée entre le président de la Société française d'archéologie et le directeur général des Beaux-arts, 30 mai 1930. 
22. Dont nous n'avons effectivement pas trace.

23. Cette vue est peut-être aussi un héritage du dessin d'architecture.

24. MAP, 80/74/3, note concernant la photographie des églises, 13 juillet 1917. Je remercie ma collègue Anne Fourestié qui m'a signalé ce document.

25. Arnaud Timbert, Restaurer et bâtir: Viollet-le-Duc en Bourgogne, Villeneuve-d'Asq, Presses universitaires du Septentrion, 2013, p. 54-58.

26. Arnaud Timbert, ibid. L'auteur se réfère à l'article d'Yves Bruand, «Église de Saint-Père sous Vézelay ", Congrès archéologique de France, Auxerre, SFA, 1958, p. 58.

27. Ces informations nous ont été communiquées par Philippe Guigon, auteur d'une communication sur «Les ruines de l'église de Lanleff » lors du Congrès archéologique de France de 2015.

\section{RÉSUMÉS}

La collection de photographies que conserve la Médiathèque de l'architecture et du patrimoine a été constituée par la commission des monuments historiques à partir de 1851. Elles ont été réalisées pour des raisons diverses : pour documenter un édifice et suivre des restaurations, des reconstructions, voire des constructions. Elles sont aussi des outils de contrôle qui permettent de juger de la pertinence d'un projet de restauration et d'apprécier les résultats obtenus. Au XIX siècle, la collaboration entre des photographes professionnels et la commission a produit des séries de prises de vues très systématiques. L'apparition d'une photographie "aide-mémoire " qui privilégie les vues de détails est liée à l'évolution du matériel photographique. Ces photographies révèlent aussi la diversité des solutions et des choix techniques des constructeurs et des restaurateurs. Confrontées aux sources textuelles et graphiques, elles peuvent inviter à une relecture de l'histoire des monuments.

MAP's collection of photographs was created by the French Commission des monuments historiques back in 1851. Those photos were taken for various reasons: the documentation of a building, restorations, reconstructions and even constructions. They act also as control tools that gauge the relevance of a restoration project while assessing its results. During the $19^{\text {th }}$ century, the bond between professional photographs and the Commission produced series of systematic shootings. The arrival of a kind of "aide-memoire" photography, that put the emphasis on detailed views, is linked with the evolution of photographic technology. These photos also reveal the variety of solutions as well as technical choices from both the builders and the restorers. They allow to have a fresh look on the history of registered historic monuments as they are confronted with graphic and textual sources.

Die in der MAP verwahrte Fotosammlung ist seit 1851 von der Commission des monuments historiques aufgebaut worden. Die Aufnahmen sind $\mathrm{zu}$ unterschiedlichem Zweck produziert worden: um einen Bau $\mathrm{zu}$ dokumentieren, um Restaurierungsarbeiten, aber auch Rekonstruktionen und Neubauten zu verfolgen; auch stellen sie ein Kontrollwerkzeug dar, das es erlaubt, die korrekte Ausführung eines Restaurierungsprojektes $\mathrm{zu}$ verfolgen und deren Ergebnisse zu bewerten. Im 19. Jahrhundert entstehen aus der Zusammenarbeit von professionellen Fotografen und der Commission eine Reihe systematischer Fotoserien. Durch die Weiterentwicklung des Materials entstehen immer mehr Detailaufnahmen, die zu einer Art 
"Merkblatt" zusammengestellt werden. Die Bestände der MAP zeugen darüber hinaus von einer großen Breite an restauratorischen und technischen Lösungen der Konservatoren und Restauratoren. Als Ergänzung $\mathrm{zu}$ schriftlichen und zeichnerischen Quellen erlauben die fotografischen Aufnahmen eine Neubewertung der Geschichte der Denkmalpflege.

\section{AUTEUR}

\section{ISABELLE GUI}

Isabelle Gui est docteur en archéologie, diplômée de l'université Paris IV. Elle a consacré sa thèse de doctorat aux « Basiliques paléochrétiennes d'Afrique du Nord». Ce travail de recherche a donné lieu à une publication sur les Basiliques chrétiennes d'Afrique du Nord (Inventaire et typologie); la première partie est parue en 1992 aux Études augustiniennes et dresse l'inventaire des monuments de l'Algérie ; la seconde partie est parue en 2014 aux éditions Ausonius et dresse cette fois l'inventaire des monuments de la Tunisie. En tant que chargée d'études documentaires, Isabelle Gui gère des collections photographiques depuis 2000 : elle a, d'abord, œuvré au Musée des arts d'Afrique et d'Océanie, puis, au Musée national des arts et traditions populaires (actuel MuCEM) et, depuis juin 2011, au département de la photographie de la Médiathèque de l'architecture et du patrimoine. Adresse électronique : isabelle.gui@culture.gouv.fr 